Documentation et bibliothèques

DOCUMENTATION BIBLIOTHEQUES

Bibliophilie contemporaine

The Contemporary Bibliophile

La bibliofilia en la actualidad

François Côté

Volume 48, numéro 1, janvier-mars 2002

URI : https://id.erudit.org/iderudit/1030468ar

DOI : https://doi.org/10.7202/1030468ar

Aller au sommaire du numéro

\section{Éditeur(s)}

Association pour l'avancement des sciences et des techniques de la documentation (ASTED)

\section{ISSN}

0315-2340 (imprimé)

2291-8949 (numérique)

Découvrir la revue

Citer cet article

Côté, F. (2002). Bibliophilie contemporaine. Documentation et bibliothèques, 48(1), 11-15. https://doi.org/10.7202/1030468ar
Résumé de l'article

Il se fait de très beaux livres au Québec, et ça ne date pas d'hier. Parallèlement au monde de l'édition prospère, celui qui s'occupe des manuels scolaires et des best-sellers que chaque éditeur espère, il y a un monde discret, celui de la bibliophilie. Il est peuplé de gens qui font du livre leur plaisir. C'est ce monde que nous allons tenter de cerner.
Tous droits réservés $@$ Association pour l'avancement des sciences et des techniques de la documentation (ASTED), 2002
Ce document est protégé par la loi sur le droit d'auteur. L'utilisation des services d'Érudit (y compris la reproduction) est assujettie à sa politique d'utilisation que vous pouvez consulter en ligne.

https://apropos.erudit.org/fr/usagers/politique-dutilisation/ 


\title{
Bibliophilie contemporaine
}

\author{
François Côté \\ Libraire
}

II se fait de très beaux livres au Québec, et ça ne date pas d'hier. Parallèlement au monde de l'édition prospère, celui qui s'occupe des manuels scolaires et des best-sellers que chaque éditeur espère, il y a un monde discret, celui de la bibliophilie. Il est peuplé de gens qui font du livre leur plaisir. C'est ce monde que nous allons tenter de cerner.

\section{The Contemporary Bibliophile \\ Québec produces some very beautiful books and has been doing so for quite some time. Parallel to the world of commercial publishing of school textbooks and best-sellers, one finds the discreet world of the bibliophile. This world is made up of people who derive pleasure from the book. This article focuses on this world.}

\begin{abstract}
La bibliofilia en la actualidad
En Quebec se producen hermosos libros, y desde hace ya mucho tiempo. A la par del mundo de la prosperidad editorial, que se ocupa de los textos escolares y los best-sellers que todo editor ansía, existe otro mundo discreto, el de la bibliofilia, habitado por aquellos que encuentran en los libros su placer. Es este universo paralelo el que intentaremos explorar.
\end{abstract}

\section{La bibliophilie}

La bibliophilie, c'est l'amour des livres. Un préjugé actuel veut que la bibliophilie concerne le livre de luxe, très dispendieux. On parle de papiers fins, d'une typographie soignée et d'estampes originales comme si rien d'autre ne pouvait exister. C'est un préjugé dû à la prédominance du livre de peintre sur le marché. Il y a maintenant toutes sortes de livres qui sont recherchés: de X-13 à Riopelle, en passant par les manuels d'économie domestique. Certains choisissent les classiques en éditions grand luxe, d'autres recherchent les populaires: romans de gare ou polars. Mais dans tous les cas, on préfère un bel exemplaire en bon état. Tant qu'à faire la dépense, mieux vaut choisir la qualité. La lecture, soit dit en passant, en est d'autant facilitée.

Intérêt, beauté, rareté: ce sont les trois préoccupations du bibliophile selon Michel Vaucaire (1970). II trace un portrait de la bibliophilie en rapport avec les champs d'intérêt des collectionneurs de livres anciens. Collectionneur est pris ici au sens noble du terme, celui qui développe une collection; on ne parlera pas ici de la " collectionnite " qui est une grave maladie (on a tous un ramasseux dans la famille). Fait intéressant, il établit un lien continuel entre la production contemporaine de livres et les champs d'intérêt historiques. Prenons l'exemple du livre pour enfants: l'explosion de la littérature jeunesse de- puis la fin de la Deuxième Guerre mondiale coïncide avec un regain d'intérêt dans la recherche, l'historiographie et le développement de collections de livres jeunesse. On pense à la comtesse de Ségur ou à Jules Vernes longtemps méprisés par les bibliophiles. Certaines de leurs éditions sont maintenant très recherchées, de même que les premières éditions de Harry Potter! Déjà.

La bibliophilie actuelle se nourrit à plusieurs sources: littéraire, technique ou artistique. En fait, tout semble maintenant permis.

\section{De la presse à bras à la rotative et à l'ordinateur}

II ne faut jamais mettre de côté les moyens de production. De la plume d'oie à la souris, les concepteurs et diffuseurs des arts du livre, ceux que j'appelle les facteurs de livres, ont toujours dû apprivoiser de nouveaux outils. À chaque bouleversement technologique, on assiste à une querelle des anciens et des modernes. Ce que nous appelons maintenant la bibliophilie traditionnelle (beau papier, belle typo et estampes originales) est apparue en réaction aux rotatives. Et la bibliophilie actuelle, on s'en doute, est en réaction à la tradition. Pourtant, les deux cohabitent à la même enseigne. Suivons le développement $\mathrm{du}$ livre illustré et de ses artisans, et voyons comment ils se transforment avec les époques.

\section{Aux environs du XVe siècle}

Il y a toujours eu des livres illustrés, même avant Gutenberg. On imprimait alors des bois gravés avec des images et du texte. C'est le cas des calendriers de bergers tirés en xylographie. En fait, avec une clientèle analphabète, il valait mieux mettre plus d'images que de textes. II nous reste en mémoire les beaux livres enluminés comme Les très Riches Heures du duc de Berry (début XV siècle).

Au milieu du $X V^{\ominus}$ siècle, l'imprimerie rendra enfin le livre accessible à toutes les bourses; mais même après cet avènement, bien des bibliophiles persévèreront à faire copier et enluminer leurs livres à la main tandis que d'autres salueront chaque innovation technologique de ce nouvel art, l'art noir, l'imprimerie ars magna. Cela durera assez longtemps, une centaine d'années, et plusieurs imprimeurs continueront d'utiliser leurs vieux caractères gothiques, en pleine Renaissance, en ayant bien soin de laisser des blancs là où pouvait s'ajouter une enluminure à la main. Mieux, on imprimait une légère gravure sur bois que le client pouvait ensuite faire colorier à moindres coûts. On avait de cette façon un livre qui ressemblait aux beaux manuscrits des seigneurs avec une calligraphie exemplaire. On poussait le luxe jusqu'à tout imprimer sur vélin. Sans 
citer de noms, rendons hommage aux autres imprimeurs-libraires qui ont su tirer parti de cette invention, peut-être en serait-on encore à faire de faux manuscrits enluminés.

La gravure sur bois va ainsi accompagner l'édition jusqu'à nos jours. Il y aura un peu de gravure sur cuivre au fil des ans, mais ce procédé en creux est difficile à adapter pour l'impression typographique qui est en relief. Cela donnera des vignettes sur bois imprimées avec le texte et des planches hors-texte tirées sur cuivre. On assemblait ensuite ces deux types de feuilles. La lithographie et la photographie n'apparaissent qu'au $\mathrm{XIX}^{\mathrm{e}}$ siècle.

\section{Au XIXe siècle}

Au XIXe, nous avons affaire à des éditeurs et non plus à des libraires-imprimeurs. L'éditeur édite, l'imprimeur imprime, le libraire vend et l'illustrateur illustre n'importe quoi: incendie, vedettes, etc., c'est son métier. Les lecteurs sont maintenant habitués aux livres illustrés. Les journaux ne sont pas en reste et on illustre l'actualité de grands bois et aussi de lithographies. Ces graveurs travaillent à la semaine ou à la pièce, ils interprètent des compositions et copient des tableaux. On ne parle pas d'artistes, au mieux, ce sont des caricaturistes comme Daumier ou Gavarni. La gravure est un art mineur, les peintres y touchent rarement.

Puis arrive la photographie. Les journaux vont faire un bond en avant avec la photo et tout un lot de graveurs-illustrateurs tombe au chômage. Les peintres-portraitistes aussi vont perdre de la clientèle. Désormais, on ira se faire tirer le portrait chez le photographe. La révolution industrielle avec ses presses à vapeur et surtout les rotatives et le papier en rouleau permet d'imprimer, en quelques minutes, ce qui prenait des semaines à produire. On peut faire plus de livres, plus vite et moins chers, mais il faut sacrifier la qualité. C'est la vie.

À cette époque, nombre de bibliophiles refusent de laisser entrer dans leur bibliothèque un ouvrage imprimé par ces machines infâmes. On soupire après les éditions des romantiques si joliment illustrées de bois gravés. On annonce la fin des arts du livre. Alors, tout naturellement apparaît le livre de bibliophilie moderne à tirage limité sur beau pápier. D'abord, dans le goût du XIXe avec de jolies lettrines (comme au
Moyen Âge), des vignettes dans le texte, des culs-de-lampe et aussi de larges compositions gravées à pleine page. Puis, de concert avec les bouleversements esthétiques de l'époque, la maquette et l'illustration du livre vont changer, mais la facture demeurera traditionnelle; il n'est pas question d'accepter de photos par exemple. Non, non, s.v.p. pas de photos!

Dans le brouhaha artistique de ce tournant de siècle, la peinture change du tout au tout; il était donc normal que l'édition de livres illustrés s'en ressente. En fait, c'est un marchand de tableaux, Ambroise Vollard, qui va imposer la contribution des peintres à l'édition. C'est en 1900 tout juste que Vollard publie Parallèlement, de Verlaine avec des lithographies originales du peintre Pierre Bonnard. II y avait eu des tentatives auparavant, mais l'histoire retient cette date. Dorénavant, nous aurons à concilier la grande tradition du livre illustré avec les audaces du livre de peintre. De l'autre côté de la Manche, il y a aussi une réaction à l'industrialisation du livre: William Morris fonde en 1890 la Kelmscott Press. II est considéré comme l'initiateur de l'important mouvement des private press dans le monde anglo-saxon.

C'est à la même période qu'on voit apparaître l'appellation estampe originale avec tout un fla-fla d'authenticité (elle sera numérotée, datée et signée). Auparavant, on pouvait voir la même gravure ici ou là, sans se préoccuper de son originalité. Maintenant, le peintre est devenu graveur et il signe son œuvre. Par cet ennoblissement, le graveur devient artiste. Les auteurs aussi vont prendre d'assaut la page imprimée. N'oublions pas qu'un manuscrit coulé dans le plomb typographique peut faire bel effet. Avec Mallarmé et Apollinaire, la mise en page devient audacieuse. Après le graveur, les autres artisans du livre - typographe, papetier et relieur réclameront leur part d'art au livre, et c'est à ce moment que tout le monde devient artiste.

\section{Au XXe siècle}

Tout le siècle est à envisager sous son rapport avec l'image, livre inclus: l'image et sa profusion sont les nouveautés. La fin du $X X^{e}$ siècle fut donc sous le signe du livre d'artiste.

On peut découper en grands pans cette histoire, somme toute récente, du livre d'artiste.
La période moderne qui va de la fin du $\mathrm{XIX}^{\mathrm{e}}$ siècle à la Deuxième Guerre mondiale comprend l'apparition de ces livres et, durant l'entre-deux guerres, son âge d'or en France.

La période contemporaine qui va de l'après-guerre à nos jours fournit une prolifération de ces ouvrages, et la commercialisation en fac-similé des grands livres des grands artistes alimente, depuis 20 ans, le débat entre «livre d'artiste » et book by artist.

Somme toute, cela fait maintenant 100 ans que l'on fait des livres d'artistes. Heureusement que d'autres éditeurs ont su tirer parti d'innovations telles que l'héliogravure et la photogravure à d'autres fins que celle de la reproduction de ces fameux grands livres.

En fait, la photographie a fait son chemin ailleurs, à part, et maintenant, avec toute une génération d'artistes multimédias, elle est mise à contribution pour malmener le livre de bibliophilie traditionnelle. Grand tirage, impression offset et prix minime, c'est la réappropriation des rotatives. C'est le phénomène du book by artist ou du book as artwork que l'on traduit toujours par le terme livre d'artiste. Du côté des auteurs, c'est le retour à l'autonomie éditoriale avec l'ordinateur et le photocopieur qui régénère le concept de private press. Rien de tel qu'un bon livre maison.

\section{Ceci est-il encore un livre?}

En janvier 2000, 14 artistes de Laval ont créé des non-livres pour une exposition à la Maison des arts de Laval. L'exposition s'intitulait Ceci n'est pas un livre. Le communiqué de presse avouait qu'un «artiste en art visuel n'est pas un écrivain". Encore un préjugé, car les écrivains ne font pas de livres, ils font des textes. II faut un coordonnateur pour faire un livre: ce paquet de feuilles reliées ou collées ensemble avec une couverture. Il y a bien un millier d'années que la forme du codex a supplanté les livres en rouleaux, volumen, et encore plus longtemps que les tablettes d'argile ont disparu. On peut maintenant parler de monographies, de manuels, de partitions ou d'atlas, ça ressemble encore et toujours à un paquet de feuilles dans lequel on trouve du texte et/ou des images et/ou de la musique! C'est un support à la diffusion d'information. La question de sa- 
voir si c'est un livre ou pas se pose, quand l'objet n'a plus l'apparence d'un paquet de feuilles...

Quelques mois plus tôt, la Bibliothèque nationale du Québec (BNQ) présentait son étonnante exposition Le scénario visuel de la page: 100 livres d'artistes. Étaient exposées une centaine d'œuvres contemporaines (1993-1997). On reconnaissait bien des livres, mais on pouvait se poser des questions sur d'autres pièces présentées. Toutes avaient en commun d'avoir été conçues par un artiste avec un lien explicite ou conceptuel au livre ou à sa manipulation, à la lecture d'informations textuelles ou iconographiques et à d'autres références, parfois ténues, ayant trait à l'écriture. Toujours est-il que la barrière qui séparait le livre (paquet de feuilles) du non-livre est tombée.

Il est amusant de noter que la Bibliothèque nationale de France présentait une exposition virtuelle à la même époque, avec le même titre (ou presque), mais qu'il s'agissait là d'une présentation historique de la mise en page typographique, donc du texte. Je rappelle que l'expression «architecture du livre" pour Marius Audin (1924) est synonyme de mise en page, alors que les artisans actuels désignent ainsi la «structure » du paquet de feuilles qu'ils tentent de «déconstruire, d'altérer ou de transgresser».

La BNQ, après avoir mis de l'avant les artistes, s'est rattrapée, en juin 2001, avec l'exposition Les Territoires extrêmes de la poésie consacrée aux auteurs. Du texte, du texte, et du texte avec quelques images au service du texte. Les précurseurs invoqués (on a tous besoin d'un grand-père) étaient, et sont toujours, Mallarmé et son Un coup de dés jamais n'abolira le hasard (1897) ainsi qu'Apollinaire et ses Calligrammes (1918). Une très bonne expo avec un échantillonnage québécois des 50 dernières années, plus deux toutes nouvelles œuvres littéraires et artistiques sur cédérom. Après les cartes perforées, floppy disk et autres disquettes harmonisées, il y a maintenant le CD comme support à l'information. Nous sommes loin du paquet de feuilles, mais comme cet attirail numérique est maintenant dans nos murs autant en profiter, quitte à élaguer les collections anciennes pour moderniser les terminaux.

\section{Étiquette et bibliothèques : questions de définitions}

Quand on se pose la question si tel ou tel objet est un encore un livre, on crée des étiquettes. Au début, vers 1960, on a taxé de livre-objet tout ce qui n'était pas conforme au tas de feuilles de papier habituel.

Naturellement, les catalogueurs se sont retrouvés devant un tas de feuilles blanches au dépôt légal et ils ont dû se demander si c'était quand même un livre ( $L e$ Livre blanc, 1976). On s'est retrouvé également avec des suites d'estampes sans texte, qui ne pouvaient certainement pas être un livre, déplorait Claudette Hould (Bibliothèque nationale du Québec 1982, 22-23) On s'est retrouvé avec un livre d'artiste dont l'emboîtage était tellement complexe que le livre est resté à la bibliothèque et l'emboîtage est allé au musée (Fragment / textes de Alain Fortaich; sérigraphies de Claude Fortaich; emboîtage de Robert Tanguay, Montréal,1985). On s'est retrouvé avec un tube rempli de pâtes alimentaires en forme de lettres de l'alphabet et on a cru à un livre à faire soi-même alors qu'il s'agissait d'une impressionnante anthologie de livres conceptuels (Bélanger, Yrénée et Guy Pressault 1974).

Depuis une vingtaine d'années, il a fallu se poser des questions pour savoir si tel livre devait se retrouver à la bibliothèque ou au musée. Du côté des bibliothèques, il est convenu que l'édition d'un livre doit comporter un texte de préférence typographié et un certain tirage (combien d'exemplaires?) À l'inverse, les musées sont prêts à accepter de la littérature sur l'art, mais pas nécessairement un livre comme œuvre d'art, à plus forte raison s'il ne contient pas d'œuvres originales. Au travers les catalogues d'expositions de bibliothèques ou de musées, les conservateurs font avancer leurs arguments en faveur de l'un ou de l'autre.

À la Bibliothèque nationale de France, lors d'une expo en 1977 (Le Livre et l'artiste, 1967-1976. Préface de Antoine Coron, conservateur à la Réserve), le préfacier insiste sur les procédés traditionnels de gravure, l'absence de photographies (réservée au livre courant) et sur son importance du point de vue littéraire. Les vedettes étaient Chagall, Ernst, Giacometti,
Miro et Picasso. Dix ans plus tard, lors d'une nouvelle exposition (50 livres illustrés depuis 1947), le même préfacier admet une crise dans l'édition du livre d'artiste. II met maintenant l'accent sur le livre entièrement réalisé et édité par un artiste et tient compte de l'amenuisement de la lisibilité du texte au profit d'un nouvel «art de voir" selon le mot du célèbre typographe lliazd. Les vedettes sont maintenant Nicolas de Staël, Jan Dubuffet et Asger Jorn ainsi que Sonia Delaunay comme précurseur, suivie de Matisse et Fautrier.

La BNQ publie son premier Répertoire des livres d'artistes au Québec, 19001980 , en 1982, sous la direction de Claudette Hould. Ce Répertoire s'inspire des grandes lignes données dans le catalogue de 1977 dirigé par A. Coron cité plus haut. Tiraillée entre notre américanité et nos racines françaises, Hould nous présente tout d'abord l'approche anglo-saxonne du book by artist avant de retenir la grande tradition française. Entre-temps est occultée la contribution du peintre Georges Delfosse au recueil d'Albert Ferland, Femmes rêvées, en 1899, parce que les dessins étaient reproduits; sont mis de côté parce que trop traditionnels, tous les bois gravés du début du siècle, incluant le Metropolitan Museum, de Robert Choquette avec bois de Edwin Holgate (1931). On trouve finalement Vitrail, de Cécile Chabot, orné d'eaux-fortes de l'auteure (1939) .conforme à la définition et Faire naître, de Roland Giguère avec les premières sérigraphies d'art dues à Albert Dumouchel (1949). Dans le dernier supplément du Répertoire de la BNQ (1999), sous la direction de Sylvie Alix, le livre d'artiste devient un livre fait par un artiste. Le Répertoire intègre ainsi plusieurs ouvrages qui n'avaient pas été retenus précédemment par manque d'originalité (!) tel Abécédaire, de Roland Giguère et Gérard Tremblay réalisé en diazocopie, blueprint, en 1975.

II y a 20 ans, plusieurs se sont demandé quand ce phénomène avait commencé. Finalement, on s'entend pour dire qu'il a pris naissance en même temps que les mouvements avant-gardistes des années 1960 avec l'acceptation de certains livres des suprématistes russes, des futuristes italiens et des dadaïstes français (il faut bien quelques précurseurs). Le point de départ serait Daily Mirror, de l'Allemand Dieter Rot (1961) et Twenty-six Gasoline Stations, de l'Américain Edward Rucha 


\section{Nomenclature en bibliophilie contemporaine}

Nous avons maintenant une nomenclature très élaborée pour cerner certains types de livres - qui font tous partie de la bibliophilie contemporaine.

1. Le livre support à l'information. Le livre normal. Qu'il soit à petit ou à grand tirage, le lecteur et le bibliophile espèrent que l'éditeur fera au mieux et que son livre sera bien fait, agréable à tenir en main et facile à lire. Avec tout l'appareillage disponible, il est aberrant de mettre sur le marché, au prix fort, un ouvrage mal imprimé et mal réglé.

Voici des définitions données d'après le concepteur original du livre. Le concepteur étant un des artisans reconnus des arts du livre.

2. Le livre d'auteur. Souvent à petit tirage, souvent de la poésie. Quand on sait que cet auteur a produit lui-même son livre, que c'est son premier ouvrage, on lui pardonne même d'avoir oublié la page de titre et, par le fait même, son nom. Touchant.

3. Le livre d'éditeur. Indépendamment du tirage, c'est lui qui est le maître d'œuvre de l'ouvrage. II retient les textes, il choisit les images. C'est le cas de la plupart des livres illustrés et des livres de peintres que l'on appelle « livres d'artistes ». Quand tout va bien, qu'un esprit de famille règne entre l'auteur, l'éditeur et l'illustrateur (artiste ou non), on court la chance d'avoir un beau livre. Autrement, on a un livre vide de sens. C'est le cas de bien des collections qui imposent un format, un «look» et même le nombre de pages! On pense à ce type d'ouvrage que l'on appelle en anglais, coffee table book

4. Le livre de graveur. Souvent à tirage restreint. Habituellement de grand format. Des estampes accompagnées d'un texte (écrit souvent par un ami). Nous avons connu au Québec une pléthore de ces très grands livres. Ce qui était tout à fait normal, compte tenu du développement des ateliers de gravure dans les années 1970-1980. Le graveur qui prend le contrôle de l'édition va faire des estampes comme auparavant il en créait pour une exposition. Il les signe et les numérote, puis il ajoute le texte et met le tout dans une boîte ou un portefeuille. C'est ce que j'appelle des expositions portatives. Il y en a des mémorables, quand même.

5. Le livre de relieur. Souvent à tirage restreint, parfois unique. II dirige l'édition et intègre son travail dans le processus de lecture. II fait en sorte que la reliure devienne un élément de la lecture.

\section{Les définitions précédentes sont issues de la tradition.}

6. Le Livre d'artiste. Nous avons assez élaboré sur le livre illustré et le livre de peintre. Nous verrons ici l'autre notion de livre d'artiste ou book by artist. On rencontre aussi les expressions Book as Artwork, Bookwork, Artists' Book et Book as Object.

Retenons comme définition générale qu'un livre d'artiste est un livre fait ou édité par un artiste. On retrouve pour les 50 dernières années autant de sous-divisions qu'il y a eu de courants ou modes en art contemporain. Voici une liste divisée en deux catégories retenue du document d'Anne Moeglin-Delcroix (1985).

Le livre comme support: livres de poésie concrète et visuelle, livres d'écriture, livres minimalistes, livres conceptuels, livres-interventions, livres de performances, livres de fiction, livres-inventaires, livres d'images, livres-journaux ou carnets de travail, livres sur le livre, livres à manipuler.

Le livre comme objet: livres condamnés, livres parasités, livres tactiles, livres manuscrits et livres peints, ceci n'est pas un livre. (Cette dernière catégorie nous ramène à cette exposition à Laval dont j'ai parlé plus haut. Sur ces livres qui sont déclarés ne pas en être, Danielle Blouin ira jusqu'à parler d'une sensation de lecture.)
(1962) (Celant 1971; Sischy 1984; Moeglin-Delcroix 1985). Au Québec, notre premier livre d'artiste contemporain est antérieur à tout cela. II s'agit de Images apprivoisées, de Roland Giguère. Montréal, Éditions Erta, 1953 (Bibliothèque nationale du Québec 1999, 22; Blouin 2001, Annexe, 153-155.)

\section{Les précurseurs contemporains}

Dans le tourbillon de l'après-guerre, il y a plus que Refus global (1948). Nous retrouvons les jolies plaquettes illustrées des Cahiers de la File indienne (19461948) dont les textes de Éloi de Grandmont, Gilles Hénault et Thérèse Renaud sont illustrés par Pellan, Daudelin et Mousseau (Yergeau 1985, 126 ; Bourassa 1977. 79). En 1949, Roland Giguère fonde les Éditions Erta qui vont s'imposer durant une vingtaine d'années grâce à la complicité de Léon Bellefleur, d'Albert Dumouchel, de Marcelle Ferron, de Claude Haeffely, de Gérard Tremblay et de bien d'autres (Grandbois 1996, 47 et suivantes). En 1958, une nouvelle génération passe à l'édition de livres illustrés. François Bujold et Guy Robert lancent les Éditions Goglin. Guy Robert continuera avec les Éditions du Songe et Iconia. Les années 1960 seront surtout littéraires avec les éditions Atys de Gilbert Langevin, Estérel de Michel Beaulieu, l'Hexagone de Gaston Miron et les Éditions d'Orphée dirigées par André Goulet. La prochaine vague de beaux livres illustrés arrive dans les années 1970. Nous retrouvons les éditions Michel Nantel et Art Global de Ara Kermoyan d'une part et, d'autre part, des publications maintenant dirigées par des artistes-graveurs comme Pierre Ayot chez Graff et Richard Lacroix à la Guilde Graphique ou les galeristes Gilles Corbeil et Gilles Gheerbrant pour ne nommer que ceux-là.

\section{Activités de bibliophilie contemporaine}

À Paris, le dernier Salon Pages \& Estampages, en novembre 2001, présentait 70 exposants, presque exclusivement des estampiers et des éditeurs d'estampes. 
À Toronto, le dernier Art of Book (1998) a reçu de 144 artistes, 323 soumissions ainsi réparties: artistes du livre $(176-54,5 \%)$, reliure artistique $(61-19 \%)$, calligraphie (30 - 9,2\%), imprimerie artistique $(29-8,75 \%)$, fabrication du papier (16 - 5\%), fabrication de boîtes (6-2\%), décoration du papier (5 - 1,5\%).

\section{Le Salon de la bibliophilie contemporaine tenu à Montréal}

À Montréal, en avril 2001 avait lieu un premier Salon de la bibliophilie contemporaine (SABIC). Ce Salon, présenté dans le cadre de la Journée mondiale du livre (lè 23 avril), était parrainé par la Confrérie de la librairie ancienne du Québec et la Bibliothèque nationale du Québec. II regroupait une quarantaine de concepteurs et diffuseurs des arts du livre que j'appelle les facteurs de livres.

Une place de choix était donnée aux micro-éditeurs actuels qui renouent parfois avec les métiers traditionnels, mais qui font souvent exploser la forme même du livre. Signalons la participation de Jacques Fournier, des Éditions Roselin, qui venait de se mériter le grand prix des Métiers d'art 2000; ainsi que celle de vétérans comme René Bonenfant des Éditions Bonfort, Pierre Filion des Éditions du Silence, Monique Dussault des Éditions du Pôle et Odette Drapeau des Éditions d'art La Tranchefile. Leurs livres sont exposés un peu partout dans le monde. L'éditeur parisien Tanguy Garric était au rendez-vous. II y avait aussi les nouveaux «gutenbergs» nés de l'ordinateur et du photocopieur. Ces nouveaux éditeurs peuvent tout réinventer de leur bureau, et leurs livres sont parfois surprenants. On a pu notamment rencontrer à ce premier Salon: l'Oie de Cravan, Mille Putois, le Relieur Fou, Steak Haché et bien d'autres.

On y trouvait également un bel éventail de la production de beaux livres des 50 dernières années, d'ici et d'ailleurs, aux stands des libraires spécialisés dont la librairie About Book de Toronto, et à ceux des galeristes Simon Blais et Eric Devlin. On a pu aussi apprécier le travail de plusieurs artisans: les typographes et pressiers Pierre Guillaume, Alain Piroir et Glenn Goluska; les relieurs d'art et restaurateurs Ghislaine Bureau, Lucie Morin et Jonathan Tremblay du groupe Les Arts du livre de Québec ainsi que Lise Dubois et Robert Jourdain de Montréal; les graveurs-éditeurs Wendy L. Simon de Canine Press, Adeline Rognon des Éditions du Rognon et plusieurs membres de XylonQuébec; la marbreure Lucie Lapierre et la calligraphe Yannick Durand de Calligrafia. II y avait des éditeurs de périodiques comme Art Le Sabord de Trois-Rivières, maintenant bien établi, et ceux du carnet de santé littéraire et graphique de la rue Ontario, Steak Haché, volontairement underground. Chacun son style. Et, dernier phénomène en liste, après les auteurs-éditeurs et les graveurs-éditeurs, voici les relieurs qui deviennent éditeurs. C'est le cas des Éditions Roselin dirigées par Jacques Fournier et des Éditions du Relieur Fou dirigées par Robert Tanguay qui ont des esthétiques diamétralement opposées, mais une commune volonté de changer la lecture par la reliure.

Ce premier Salon fait date. II a permis aux participants de se rencontrer, d'échanger et de présenter leurs livres à un millier de visiteurs venus découvrir cette production. Ces livres, séduisants ou inusités, ont une caractéristique commune : leur manque de diffusion. Ne les cherchez pas en librairies, ils ne sont pas dans le réseau. On peut quand même en voir dans quelques galeries d'art et dans certaines librairies spécialisées. II faut dire que ces éditions, souvent artisanales, sont réalisées à petits tirages qu'il faut commander directement chez l'auteur, l'éditeur ou l'artiste. On aime bien voir avant d'acquérir, mais avant d'acquérir, il faut désirer, et comment peut-on désirer quelque chose dont on ne soupçonne même pas l'existence.

Le deuxième Salon de la bibliophilie contemporaine s'est tenu le 21 avril 2002, il mettait l'accent sur la micro-édition, traditionnelle et contemporaine, sélecte et underground.

\section{Sources consultées}

Audin, Marius. 1942. Le Livre, Son architecture, Sa technique. Paris: Crès.

Bélanger, Yrénée et Guy Pressault. 1974. Des mêmes auteurs. [Ottawa], Éditions de l'œuf.

Bibliothèque nationale de France. 1977. Le Livre et l'artiste, 1967-1976. Sous la direction de Antoine Coron. Paris. 1988. 50 livres illustrés depuis 1947. Sous la direction de Antoine Coron. Paris.

Bibliothèque nationale du Québec. 1982. Répertoire des livres d'artistes au Québec, 1900-1980. Sous la direction de Claudette Hould. Montréal.

1999. Répertoire des livres d'artistes au Québec 1993-1997. Sous la direction de Sylvie Alix. Montréal.

Blouin, Danielle. 2001. Un livre délinquant. Montréal : Fides.

Bourassa, André-G. 1977. Surréalisme et littérature québécoise. L'Étincelle.

Celant, German. 1971. Le Livre comme œuvre d'art 1960-1972. Genoa. Trad. par René Blouin et Elisa Bonvici. 1981. In Book by artists. Toronto, Art Metropole.

Grandbois, Michèle. 1996. L'Art québécois de l'estampe. Québec, Musée du Québec.

Le Livre blanc. 1976. Montréal: Éditions internationales Alain Stanké.

Moeglin-Deicroix, Anne. 1985. Livres d'artistes. Paris: Pompidou-Hersher.

Sischy. Ingrid. 1984. Printed Matter Catalog 1983/84, Book by artists. NY: Printed Matter.

Vaucaire, Michel. 1970. La bibliophilie. Paris : PUF. (Que sais-je? 1406).

Yergeau, Robert.1985. In: L'Édition littéraire au Québec de 1940 à 1960. Sherbrooke : Université de Sherbrooke, Département d'études françaises, Faculté des arts. 